\title{
A NOTE ON MEASURE HOMOLOGY
}

\author{
ROBERTO FRIGERIO \\ Dipartimento di Matematica Università di Pisa Largo B. Pontecorvo 5, \\ Pisa 56127, Italy \\ e-mail:frigerio@dm.unipi.it
}

(Received 3 May 2012; revised 3 September 2012; accepted 24 January 2013; first published online 13 August 2013)

\begin{abstract}
Measure homology was introduced by Thurston (W. P. Thurston, The geometry and topology of 3-manifolds, mimeographed notes (Princeton University Press, Princeton, NJ, 1979)) in order to compute the simplicial volume of hyperbolic manifolds. Berlanga (R. Berlanga, A topologised measure homology, Glasg. Math. J. 50 (2008), 359-369) endowed measure homology with the structure of a graded, locally convex (possibly non-Hausdorff) topological vector space. In this paper we completely characterize Berlanga's topology on measure homology of CW-complexes, showing in particular that it is Hausdorff. This answers a question posed by Berlanga.
\end{abstract}

2000 Mathematics Subject Classification. 55N35.

1. Introduction. Measure homology was introduced by Thurston in [10], where it was exploited to compute the simplicial volume of closed hyperbolic manifolds. It is proved in $[\mathbf{5}, \mathbf{1 1}]$ that measure homology is canonically isomorphic to the usual real singular homology, at least for CW-complexes. Moreover, Loeh proved in [7] that these homology theories are not only isomorphic but also isometric (with respect to Thurston's seminorm on measure homology [10] and Gromov's seminorm on singular homology [4]), a fact that plays a fundamental rôle in applications to the simplicial volume. For a comprehensive account about the notion of measure homology and its applications, see e.g. the Introductions of $[\mathbf{1 1}, \mathbf{1}]$.

Thurston's seminorm is not the only extra-structure naturally supported by measure homology. In [1], for every $n \in \mathbb{N}$ the $n$th measure homology module $\mathcal{H}_{n}(X)$ of a topological space $X$ is endowed with a natural structure of a locally convex (possibly non-Hausdorff) topological vector space.

Let us now recall the main result of [1]

THEOREM 1.1. Suppose that $X$ has the homotopy type of a countable $C W$-complex. Then $\mathcal{H}_{1}(X)$ is a locally convex Hausdorff vector space.

In [1] Berlanga asks the following:

QUESTION 1.2. Is $\mathcal{H}_{n}(X)$ a Hausdorff space in general?

In this paper we completely characterize Berlanga's topology on measure homology, giving in particular a positive answer to Question 1.2, at least in the case of CW-complexes. If $W$ is a real vector space, then the strongest weak topology on $W$ is the weakest topology which makes every linear functional on $W$ continuous (see Section 2.3). Our main result is the following. 
Theorem 1.3. Suppose that $X$ has the homotopy type of a $C W$-complex and let $n \in \mathbb{N}$. Then Berlanga's topology on $\mathcal{H}_{n}(X)$ coincides with the strongest weak topology. In particular, it is Hausdorff.

Let us briefly discuss the meaning of Theorem 1.3. In order to study the isomorphism and the isometry type of homology modules, it is often useful to take advantage of suitable duality principles that reduce computations in homology to computations in cohomology. This strategy, which in the context of singular homology dates back to Gromov [4], has been profitably put to use also in the study of measure homology and $\ell^{1}$-homology (see e.g. $[7,8]$ ). Theorem 1.3 may be rephrased by saying that the topological dual space of measure homology coincides with its abstract dual space, and this fact may be probably exploited to provide effective duality arguments in the study of measure homology.

On the other hand, Theorem 1.3 also has some disappointing consequences. For example, it implies that, at least for CW-complexes, the isomorphism type of measure homology as a graded real vector space (which coincides in turn with the isomorphism type of the corresponding ordinary singular homology $[\mathbf{5}, \mathbf{1 1}]$ ) completely determines the isomorphism type of measure homology as a graded topological vector space. In particular, the additional structure provided by Berlanga's topology cannot be used to distinguish CW-complexes sharing the same singular homology. Moreover, as proved in Remark 3.2 at the end of the paper, Berlanga's topology is not related to the topology induced by Thurston's seminorm on measure homology. As a consequence, Theorem 1.3 seems to suggest that Berlanga's topology may intervene in applications to the simplicial volume just via the duality arguments mentioned above.

\section{Preliminaries.}

2.1. Measure homology. Let $X$ be a topological space and let $S_{n}(X)$ be the set of singular $n$-simplices with values in $X$. We endow $S_{n}(X)$ with the compact-open topology and denote by $\mathbb{B}_{n}(X)$ the $\sigma$-algebra of Borel subsets of $S_{n}(X)$. If $\mu$ is a signed measure on $\mathbb{B}_{n}(X)$ (in this case we say for short that $\mu$ is a Borel measure on $S_{n}(X)$ ), then $\mu$ has finite total variation if $|\mu(A)|<\infty$ for every $A \in \mathbb{B}_{n}(X)$. For every $n \geq 0$, the measure chain module $\mathcal{C}_{n}(X)$ is the real vector space of the Borel measures on $S_{n}(X)$ having finite total variation and admitting a compact determination set (see [11] for the definition of determination set and a detailed discussion of the relationship between this notion and the notion of support). The graded module $\mathcal{C}_{*}(X)$ can be given the structure of a complex via the boundary operator

$$
\partial_{n}: \mathcal{C}_{n}(X) \rightarrow \mathcal{C}_{n-1}(X), \quad \partial_{n} \mu=\sum_{j=0}^{n}(-1)^{j} \mu^{j}
$$

where $\mu^{j}$ is the push-forward of $\mu$ under the map that takes a simplex $\sigma \in S_{n}(X)$ into the composition of $\sigma$ with the usual inclusion of the standard $(n-1)$-simplex onto the $j$ th face of $\sigma$. The homology of the complex $\left(\mathcal{C}_{*}(X), \partial_{*}\right)$ is the measure homology of $X$, and it is denoted by $\mathcal{H}_{*}(X)$. 
2.2. Berlanga's topology. If $\lambda: S_{n}(X) \rightarrow \mathbb{R}$ is a continuous function, then the map

$$
\psi_{\lambda}: \mathcal{C}_{n}(X) \rightarrow \mathbb{R}, \quad \mu \mapsto \int_{S_{n}(X)} \lambda \mathrm{d} \mu
$$

is a well-defined linear functional on $\mathcal{C}_{n}(X)$. Following [1] we put on $\mathcal{C}_{n}(X)$ the weakest topology which makes $\psi_{\lambda}$ continuous for every continuous $\lambda: S_{n}(X) \rightarrow \mathbb{R}$. We also put on $\mathcal{H}_{n}(X)$ the quotient topology induced by the restriction of the topology of $\mathcal{C}_{n}(X)$ to the subspace ker $\partial_{n} \subseteq \mathcal{C}_{n}(X)$ of measure cycles.

2.3. Topological vector spaces. By a topological vector space we mean a real vector space endowed with a topology $\tau$ such that the vector space operations are continuous with respect to $\tau$ (in particular, we do not require that $\tau$ is Hausdorff). It is readily seen that Berlanga's topology endows $\mathcal{C}_{n}(X)$ with the structure of a locally convex topological vector space. Since local convexity is inherited by subspaces and quotients, the vector space $\mathcal{H}_{n}(X)$ is a locally convex (possibly non-Hausdorff) topological vector space.

Let us now put Berlanga's definition into the general context of weak topologies associated to pairings. If $V, W$ are real vector spaces, a pairing between $V$ and $W$ is simply a bilinear map $\eta: V \times W \rightarrow \mathbb{R}$. Let $W^{*}$ be the algebraic dual of $W$. We say that an element $\beta \in W^{*}$ is represented by $v \in V$ via $\eta$ if $\beta=\eta(v, \cdot)$, and we denote by $W^{*}(\eta) \subseteq W^{*}$ the subset of functionals that are represented via $\eta$ by some element of $V$. The weak topology on $W$ corresponding to $\eta$ is the weakest topology which makes every element of $W^{*}(\eta)$ continuous. This topology endows $W$ with the structure of a locally convex topological vector space, and it is Hausdorff if and only if for every $w \in W$ there exists $v \in V$ such that $\eta(v, w) \neq 0$ (see e.g. [6, Section 16]).

We define the strongest weak topology $\tau_{\mathrm{sw}}^{W}$ on $W$ as the weak topology associated to any pairing $\eta$ such that $W^{*}(\eta)=W^{*}$. So $\tau_{\mathrm{sw}}^{W}$ endows $W$ with the structure of a locally convex Hausdorff vector space.

REMARK 2.1. Let $\tau_{\text {slc }}^{W}$ be the strongest locally convex topology on $W$ (see e.g. [6] for the definition and several properties of $\tau_{\mathrm{slc}}^{W}$ ). If $W$ is finite-dimensional, then $\tau_{\mathrm{sw}}^{W}=\tau_{\mathrm{slc}}^{W}$, and they both coincide with the Euclidean topology on $W$. Otherwise, $\tau_{\mathrm{sw}}^{W}$ is strictly weaker than $\tau_{\mathrm{slc}}^{W}$. In fact, let $p: W \rightarrow \mathbb{R}$ be a norm. The unit ball of $p$ does not contain any non-trivial linear subspace of $W$, while every $\tau_{\mathrm{sw}}^{W}$-neighbourhood of $0 \in W$ contains a finite co-dimensional linear subspace of $W$. Therefore, if $\operatorname{dim} W=\infty$ then $p$ is not continuous with respect to $\tau_{\mathrm{sw}}^{W}$. On the other hand, every norm is continuous with respect to $\tau_{\mathrm{slc}}^{W}$, so $\tau_{\mathrm{sw}}^{W} \neq \tau_{\mathrm{slc}}^{W}$ unless the dimension of $W$ is finite.

2.4. Singular homology vs. measure homology. Let $X$ be a topological space. We denote by $C_{*}(X)$ the complex of real singular chains of $X$, and by $C^{*}(X)$ the complex of real singular cochains of $X$, i.e. the algebraic dual complex of $C_{*}(X)$. We also let $H_{*}(X)$ (resp. $\left.H^{*}(X)\right)$ be the homology of the complex $C_{*}(X)\left(\operatorname{resp} . C^{*}(X)\right)$, i.e. the usual real singular homology (resp. cohomology) of $X$. For every $\sigma \in S_{n}(X), n \in \mathbb{N}$, let us denote by $\delta_{\sigma} \in \mathcal{C}_{n}(X)$ the atomic measure supported by the singleton $\{\sigma\}$. The 
chain map

$$
\iota_{*}: C_{*}(X) \rightarrow \mathcal{C}_{*}(X), \quad \iota_{*}\left(\sum_{i=0}^{k} a_{i} \sigma_{i}\right)=\sum_{i=0}^{k} a_{i} \delta_{\sigma_{i}},
$$

induces a map

$$
H_{*}\left(\iota_{*}\right): H_{*}(X) \longrightarrow \mathcal{H}_{*}(X)
$$

The following result was proved independently by Zastrow [11] and Hansen [5]:

THEOREM $2.2[5, \mathbf{1 1}]$. Suppose that $X$ has the homotopy type of a $C W$-complex. Then the map

$$
H_{n}\left(\iota_{n}\right): H_{n}(X) \longrightarrow \mathcal{H}_{n}(X)
$$

is an algebraic isomorphism for every $n \in \mathbb{N}$.

2.5. Continuous cohomology. Let us now recall the definition of continuous cohomology of a topological space $X$. We regard $S_{n}(X)$ as a subset of $C_{n}(X)$ so that for every cochain $\varphi \in C^{n}(X)$ it makes sense to consider the restriction $\left.\varphi\right|_{S_{n}(X)}$. We say that $\varphi$ is continuous if $\left.\varphi\right|_{S_{n}(X)}$ is, and we set

$$
C_{c}^{*}(X)=\left\{\varphi \in C^{*}(X) \mid \varphi \text { is continuous }\right\} .
$$

It is readily seen that $C_{c}^{*}(X)$ is a subcomplex of $C^{*}(X)$. Its homology is the continuous cohomology of $X$, and it is denoted by $H_{c}^{*}(X)$. The following result describes the relationship between continuous cohomology and the usual singular cohomology.

TheOREM 2.3. Suppose that $X$ has the homotopy type of a $C W$-complex. Then the inclusion $\rho^{*}: C_{c}^{*}(X) \hookrightarrow C^{*}(X)$ induces isomorphisms

$$
H^{n}\left(\rho^{n}\right): H_{c}^{n}(X) \rightarrow H^{n}(X), \quad n \in \mathbb{N}
$$

Proof. By [9, Theorem 2], any CW-complex has the homotopy type of a simplicial complex, endowed with the metric topology in the sense of Eilenberg and Steenrod [2, p. 75]. Therefore, $X$ has the homotopy type of a locally contractible metrizable space, and the conclusion follows from [3, Theorem 1.1].

3. Proof of theorem 1.3. Let $X$ be a topological space. For every $n \in \mathbb{N}$, the map

$$
C_{c}^{n}(X) \times \mathcal{C}_{n}(X) \rightarrow \mathbb{R}, \quad(\varphi, \mu) \mapsto \int_{S_{n}(X)} \varphi \mathrm{d} \mu
$$

induces a pairing

$$
\eta_{n}: H_{c}^{n}(X) \times \mathcal{H}_{n}(X) \rightarrow \mathbb{R}
$$


It is very easy to compare this pairing with the usual Kronecker pairing

$$
\kappa_{n}: H^{n}(X) \times H_{n}(X) \rightarrow \mathbb{R}
$$

between singular homology and singular cohomology. In fact, it readily follows from the definitions that

$$
\eta_{n}\left(\alpha, H_{n}\left(\iota_{n}\right)(\beta)\right)=\kappa_{n}\left(H^{n}\left(\rho^{n}\right)(\alpha), \beta\right) \quad \text { for every } \alpha \in H_{c}^{n}(X), \beta \in H_{n}(X) .
$$

By construction, Berlanga's topology on $\mathcal{H}_{n}(X)$ coincides with the weak topology associated to the pairing $\eta_{n}$. Therefore, Theorem 1.3 may be restated as follows:

THEOREM 3.1. Suppose that $X$ has the homotopy type of a $C W$-complex. Then every linear functional on $\mathcal{H}_{n}(X)$ is represented via $\eta_{n}$ by some element in $H_{c}^{n}(X)$.

Proof. Let $\beta: \mathcal{H}_{n}(X) \rightarrow \mathbb{R}$ be a fixed linear functional. The Universal Coefficient Theorem ensures that the composition $\beta \circ H_{n}\left(\iota_{n}\right): H_{n}(X) \rightarrow \mathbb{R}$ is represented via $\kappa_{n}$ by an element in $H^{n}(X)$, i.e. there exists $\gamma \in H^{n}(X)$ such that

$$
\kappa_{n}(\gamma, c)=\beta\left(H_{n}\left(\iota_{n}\right)(c)\right) \text { for every } c \in H_{n}(X) .
$$

Recall now from Theorem 2.3 that the map $H^{n}\left(\rho^{n}\right): H_{c}^{n}(X) \rightarrow H^{n}(X)$ is an isomorphism, and set $\gamma_{c}=H^{n}\left(\rho^{n}\right)^{-1}(\gamma) \in H_{c}^{n}(X)$. From equations (1) and (2) we deduce that

$$
\eta_{n}\left(\gamma_{c}, H_{n}\left(\iota_{n}\right)(c)\right)=\kappa_{n}(\gamma, c)=\beta\left(H_{n}\left(\iota_{n}\right)(c)\right) \text { for every } c \in H_{n}(X) .
$$

By Theorem 2.2, the map $H_{n}\left(\iota_{n}\right): H_{n}(X) \rightarrow \mathcal{H}_{n}(X)$ is an isomorphism, so the last equation implies that $\eta_{n}\left(\gamma_{c}, \cdot\right)=\beta$ on the whole of $\mathcal{H}_{n}(X)$. We have thus shown that $\beta$ is represented by $\gamma_{c}$ via $\eta_{n}$, and this concludes the proof.

We conclude the paper with the following remark that describes the (lack of) relationship between Berlanga's topology and the topology induced by Thurston's seminorm on measure homology.

Remark 3.2. Let $X$ be a CW-complex, and let us call Thurston's topology the locally convex topology on $\mathcal{H}_{n}(X)$ associated to Thurston's seminorm. We show here that there are examples where Thurston's topology is not finer than Berlanga's one, and vice versa.

Recall that the simplicial volume of the closed orientable surface $\Sigma_{2}$ of genus two is positive $[4,10]$. Therefore, if $X_{\infty}$ is the disjoint union of a countable family of copies of $\Sigma_{2}$, then it is easily checked that Gromov's seminorm on $H_{2}\left(X_{\infty}\right)$ is in fact a norm. Since singular homology and measure homology are isometrically isomorphic [7], the same is true for Thurston's seminorm on $\mathcal{H}_{2}\left(X_{\infty}\right)$. Since $\operatorname{dim} H_{2}\left(X_{\infty}\right)=\infty$, the argument described in Remark 2.1 shows that in this case Berlanga's topology is not finer than Thurston's one. On the other hand, every normed infinite dimensional vector space admits a non-continuous linear functional, and this implies that Thurston's topology on $\mathcal{H}_{2}\left(X_{\infty}\right)$ is not finer than Berlanga's one.

If $\operatorname{dim} \mathcal{H}_{n}(X)<\infty$, then Berlanga's topology is the strongest locally convex topology on $\mathcal{H}_{n}(X)$, and is therefore finer than Thurston's one. More precisely, in the finite dimensional case, Berlanga's topology is strictly finer than Thurston's one if and only if Thurston's seminorm is not a norm (this is the case, for example, for $\left.\mathcal{H}_{1}\left(S^{1}\right)\right)$. 


\section{ROBERTO FRIGERIO}

\section{REFERENCES}

1. R. Berlanga, A topologised measure homology, Glasg. Math. J. 50 (2008), 359-369.

2. S. Eilenberg and N. E. Steenrod, Foundations of algebraic topology (Princeton University Press, Princeton, NJ, 1952).

3. R. Frigerio, (Bounded) continuous cohomology and Gromov's proportionality principle, Manuscripta Math. 134 (2011), 435-474.

4. M. Gromov, Volume and bounded cohomology, Inst. Hautes Études Sci. Publ. Math. 56 (1982), 5-99.

5. S. K. Hansen, Measure homology, Math. Scand. 83 (1998), 205-219.

6. J. L. Kelley and I. Namioka, Linear topological spaces, Graduate Texts in Mathematics, No. 36 (Springer-Verlag, New York, NY, 1976).

7. C. Löh, Measure homology and singular homology are isometrically isomorphic, Math. Z. 253 (2006), 197-218.

8. C. Löh, Isomorphisms in $l^{1}$-homology, Münster J. Math. 1 (2008), 237-266.

9. J. Milnor, On spaces having the homotopy type of a CW-complex, Trans. Amer. Math. Soc. 90 (1959), 272-280.

10. W. P. Thurston, The geometry and topology of 3-manifolds, mimeographed notes (Princeton University Press, Princeton, NJ, 1979).

11. A. Zastrow, On the (non)-coincidence of Milnor-Thurston homology theory with singular homology theory, Pacific J. Math. (1998), 186(2), 369-396. 